Electronic Supplementary Information

for

\title{
Biomass derived high areal and specific capacity hard carbon anodes for sodium-ion batteries
}

\author{
Uttam Kumar ${ }^{*+}$, Jimmy $\mathbf{W u}^{\ddagger}$, Neeraj Sharma ${ }^{\ddagger}$, Veena Sahajwalla ${ }^{*+}$ \\ ${ }^{\dagger}$ Centre for Sustainable Materials Research and Technology (SMaRT@UNSW), \\ School of Materials Science and Engineering, UNSW Sydney, NSW 2052, Australia \\ ‡ School of Chemistry, UNSW Sydney, NSW 2052, Australia \\ *Corresponding author: (uttam.kumar@unsw.edu.au and veena@unsw.edu.au)
}




\section{Macshell}

Carbonisation

$700-1100{ }^{\circ} \mathrm{C}$

$\mathrm{N}_{2}, 1 \mathrm{~h}$

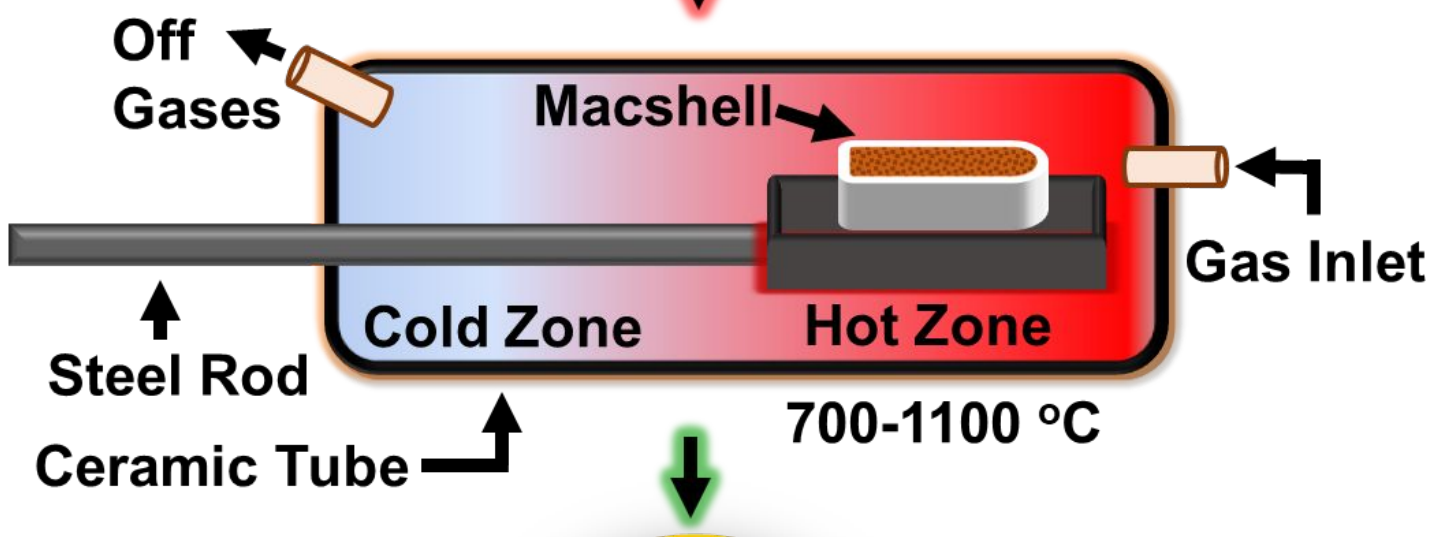

\section{Hard}

Carbon

Figure S1. Schematic of the furnace used to synthesize M7, M9, and M11 hard carbons. 
Table S1. XRF analysis of the raw macshell sample.

\begin{tabular}{ll}
\hline Oxide & Wt.\% \\
\hline $\mathrm{Na}_{2} \mathrm{O}$ & 5.30 \\
\hline $\mathrm{MgO}$ & 7.34 \\
\hline $\mathrm{Al}_{2} \mathrm{O}_{3}$ & 1.34 \\
\hline $\mathrm{SiO}_{2}$ & 1.25 \\
\hline $\mathrm{P}_{2} \mathrm{O}_{5}$ & 6.94 \\
\hline $\mathrm{SO}_{3}$ & 6.75 \\
\hline $\mathrm{K}_{2} \mathrm{O}$ & 43.27 \\
\hline $\mathrm{CaO}$ & 9.58 \\
\hline $\mathrm{Mn}_{3} \mathrm{O}_{4}$ & 1.23 \\
\hline $\mathrm{Fe}_{2} \mathrm{O}_{3}$ & 0.94 \\
\hline $\mathrm{CuO}$ & 0.22 \\
\hline $\mathrm{ZnO}$ & 0.14 \\
\hline $\mathrm{BaO}$ & 0.04 \\
\hline $\mathrm{SnO}$ & 0.22 \\
\hline $\mathrm{L.O} . \mathrm{I}$. & 12.37 \\
\hline
\end{tabular}



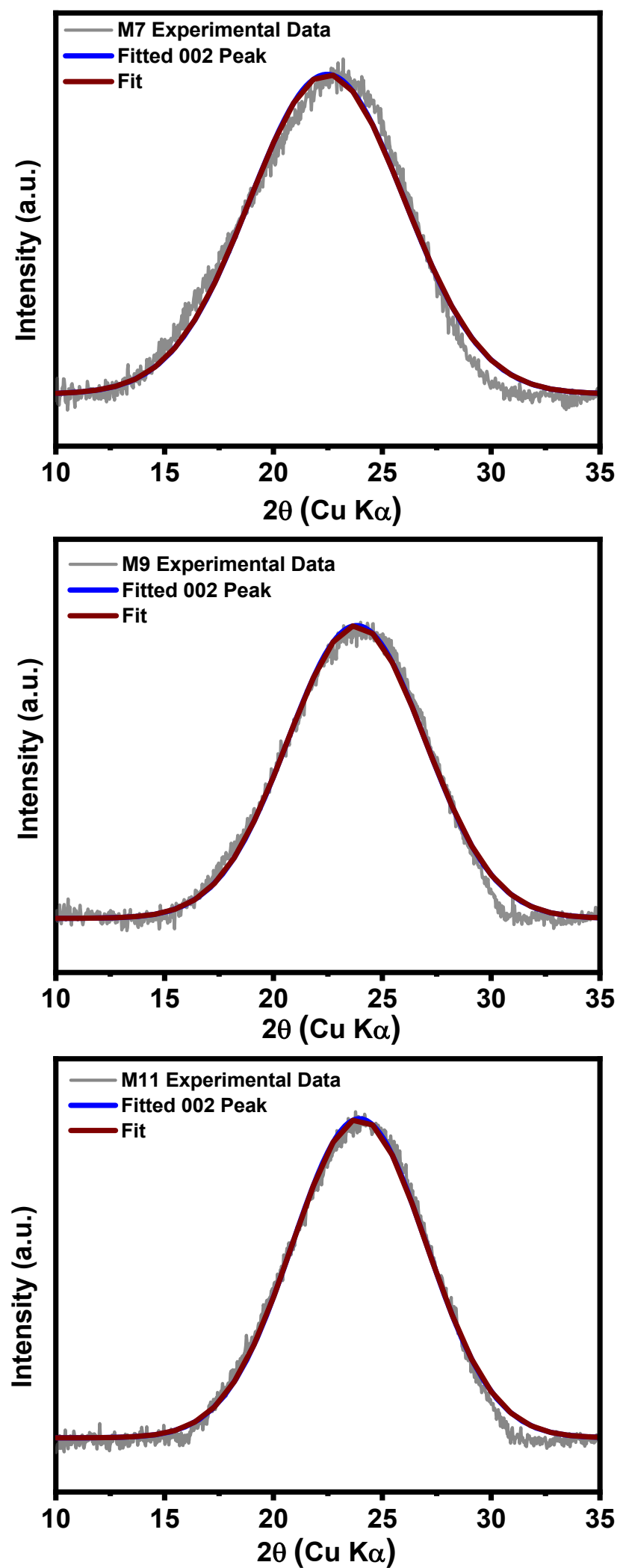

Figure S2: Gaussian fit for the XRD (002) peak for M7, M9, and M11. 
Table S2: Peak positions and atomic \% of fitted XPS peaks for M7, M9, and M11 samples.

\begin{tabular}{|c|c|c|c|c|c|c|}
\hline \multirow[b]{2}{*}{ Name } & \multicolumn{2}{|c|}{ M7 } & \multicolumn{2}{|c|}{ M9 } & \multicolumn{2}{|c|}{ M11 } \\
\hline & Peak BE (eV) & Atomic \% & Peak BE (eV) & Atomic \% & Peak BE (eV) & Atomic \% \\
\hline C1s A & 284.5 & 62.33 & 284.5 & 56.45 & 284.5 & 53.90 \\
\hline C1s B & 285.3 & 7.69 & 285.3 & 7.88 & 285.3 & 9.23 \\
\hline $\mathrm{C} 1 \mathrm{~s} \mathrm{C}$ & 286.1 & 7.71 & 286.1 & 9.14 & 286.1 & 8.33 \\
\hline C1s D & 287.5 & 4.73 & 287.5 & 5.27 & 287.5 & 5.20 \\
\hline C1s E & 289.1 & 3.93 & 289.1 & 4.54 & 289.1 & 4.40 \\
\hline C1s F & 290.9 & 2.21 & 290.8 & 3.25 & 290.8 & 3.27 \\
\hline K2p3 A & 293.2 & 1.10 & 293.3 & 2.44 & 293.3 & 3.09 \\
\hline O1s A & 532.1 & 3.87 & 533.6 & 2.73 & 533.6 & 2.37 \\
\hline O1s B & 533.5 & 3.56 & 532.2 & 4.10 & 532.3 & 4.29 \\
\hline O1s C & 530.8 & 1.02 & 530.9 & 1.17 & 530.9 & 2.00 \\
\hline F1s A & 687.0 & 1.05 & 687.1 & 1.08 & 687.1 & 1.19 \\
\hline $\begin{array}{l}\mathrm{Cl} 2 \mathrm{p} 3 \\
\mathrm{~A}\end{array}$ & 198.6 & 0.52 & 198.7 & 1.01 & 198.7 & 1.62 \\
\hline $\begin{array}{l}\text { Mg1s } \\
\text { A }\end{array}$ & - & - & 1305.0 & 0.36 & 1305.2 & 0.67 \\
\hline Si2p A & 102.1 & 0.28 & 102.1 & 0.32 & 102.2 & 0.26 \\
\hline P2p A & - & - & 136.7 & 0.15 & 136.5 & 0.11 \\
\hline P2p B & - & - & 133.9 & 0.12 & 133.9 & 0.06 \\
\hline
\end{tabular}




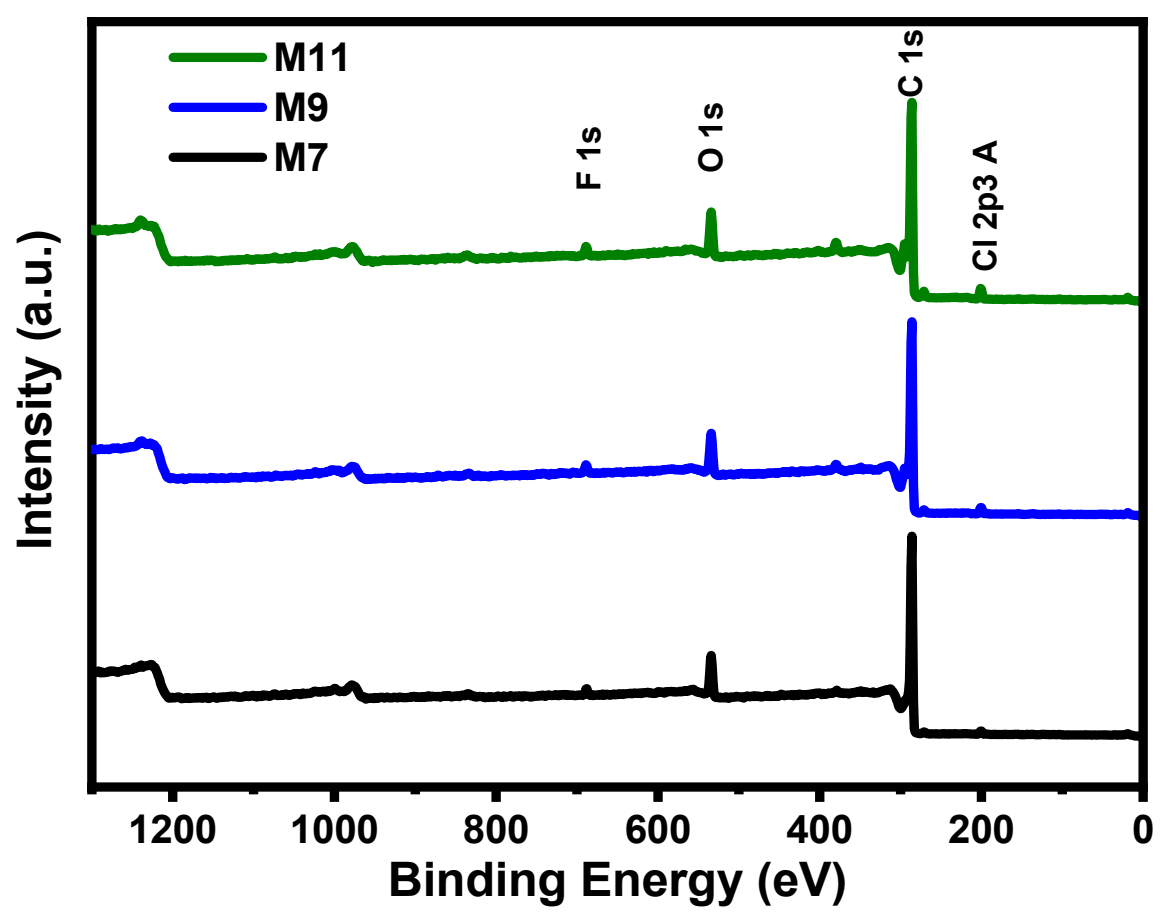

Figure S3. XPS survey Scan for M7, M9, and M11.
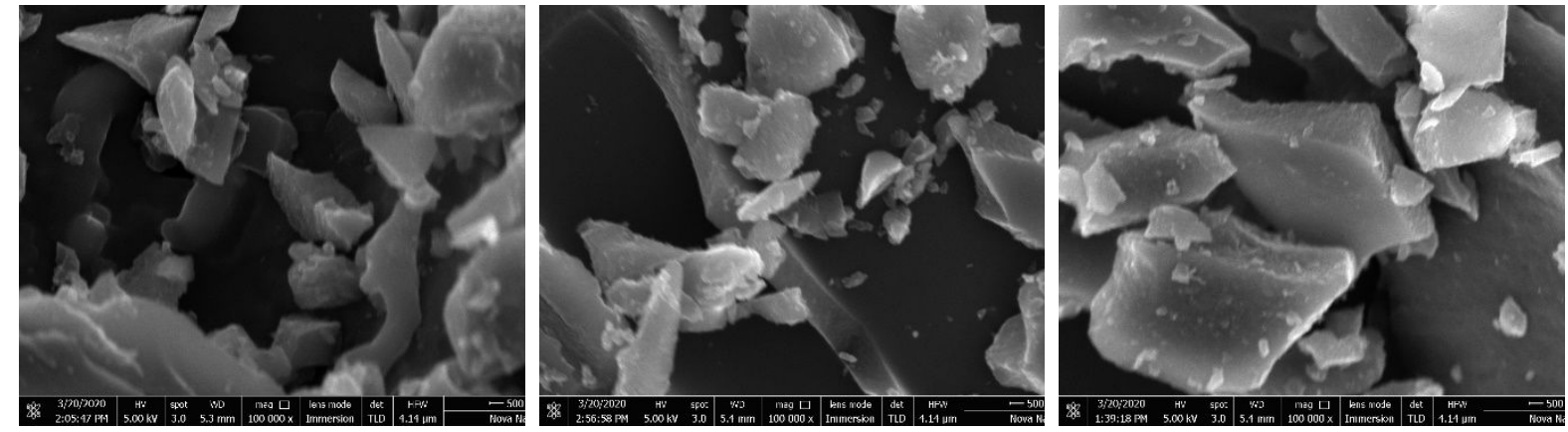

Figure S4. SEM image (obtained using Nova NanoSEM 450) for M7, M9, and M11 at a magnification of 100000x. 
Table S3: Particle size distribution for M11 hard carbon.

\begin{tabular}{cccc}
\hline Record Number & Dx (10) $(\boldsymbol{\mu m})$ & Dx (50) $(\boldsymbol{\mu m})$ & Dx (90) $(\boldsymbol{\mu m})$ \\
\hline 1 & 4.63 & 23.9 & 97.1 \\
2 & 4.62 & 23.8 & 95.4 \\
3 & 4.63 & 24.0 & 96.0 \\
\hline Mean & 4.63 & 23.9 & 96.1 \\
\hline Std Dev & 0.00730 & 0.0980 & 0.867 \\
\hline
\end{tabular}

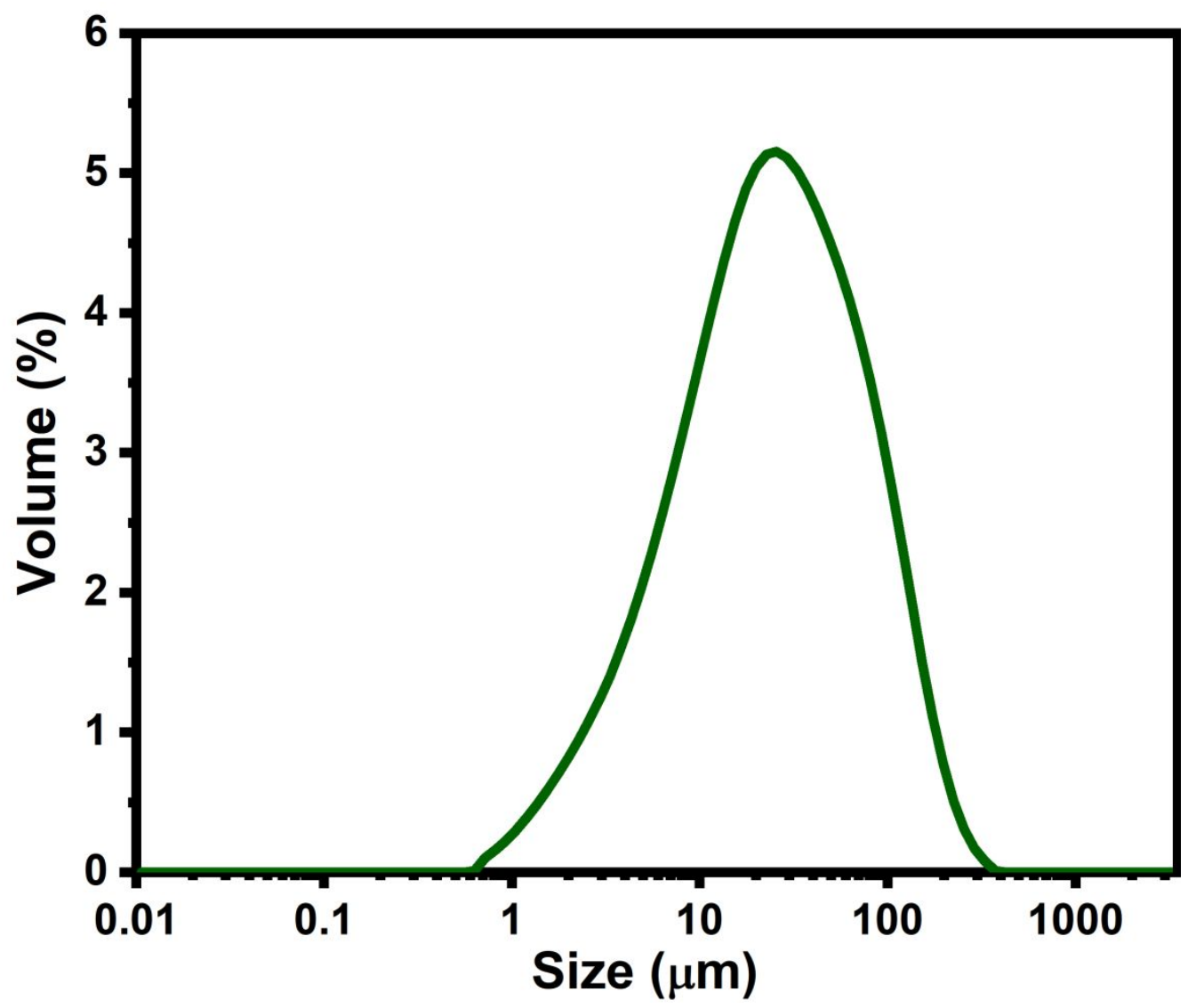

Figure S5. Particle size distribution for the M11 hard carbon. The data on x-axis is plotted on a logarithmic scale. 

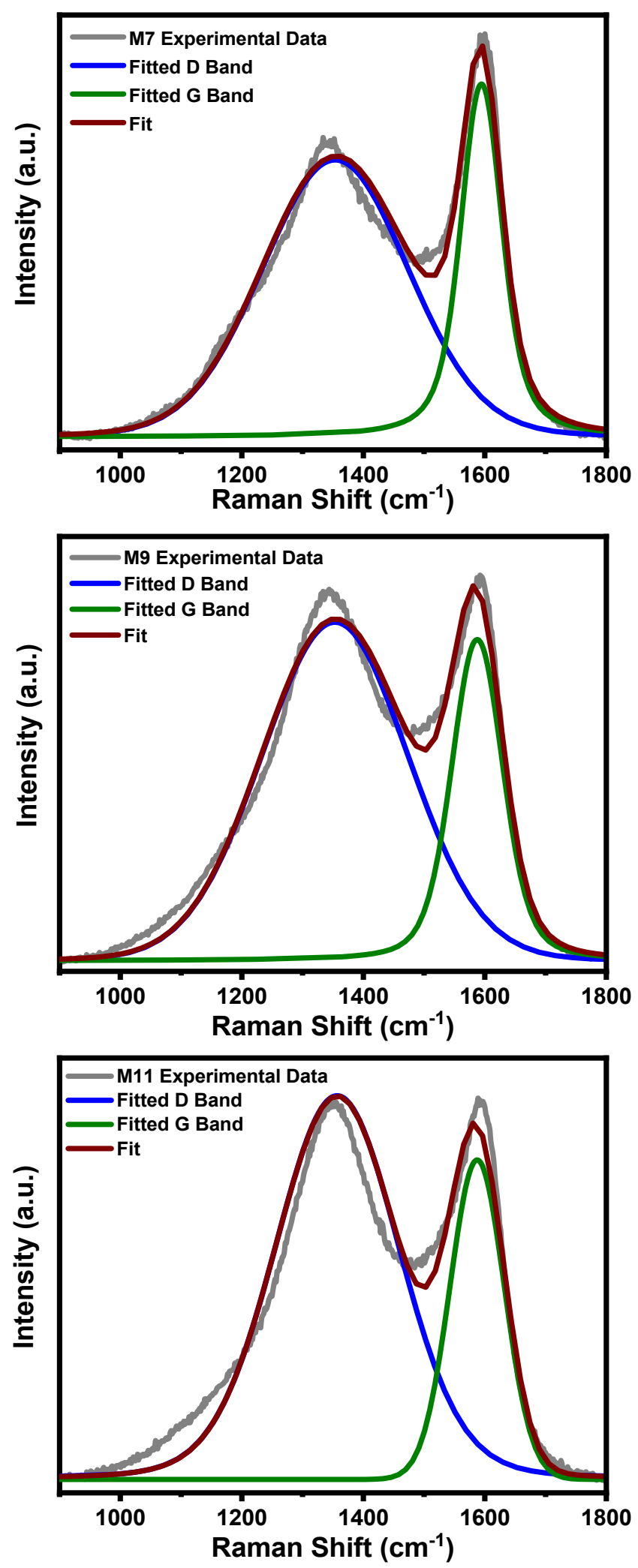

Figure S6: Curve fitted D and G bands for Raman spectra for M7, M9, and M11. 
Table S4. Comparison of the performance of the carbons reported in literature versus the carbons used in this work for NIBs.

\begin{tabular}{|c|c|c|c|c|c|c|}
\hline \multirow{2}{*}{$\begin{array}{l}\text { Precursor } \\
\text { material }\end{array}$} & \multirow{2}{*}{$\begin{array}{c}\text { Synthesis } \\
\text { temperature } \\
\left({ }^{\circ} \mathrm{C}\right)\end{array}$} & \multirow{2}{*}{$\begin{array}{l}\text { Current } \\
\text { density } \\
(\mathrm{mA} / \mathrm{g})\end{array}$} & \multicolumn{2}{|c|}{ Cyclability } & \multirow{2}{*}{$\begin{array}{c}\text { Additional } \\
\text { Treatments/purification of } \\
\text { synthesised carbon }\end{array}$} & \multirow[t]{2}{*}{ Ref. } \\
\hline & & & Cycle & $\begin{array}{c}\text { Reversible } \\
\text { capacity } \\
\text { (mAh/g) }\end{array}$ & & \\
\hline $\begin{array}{l}\text { Carbotron } \\
\text { (commercial } \\
\text { carbon) }\end{array}$ & - & 250 & 300 & 220 & - & 1 \\
\hline Olive Stones & 1000 & 20 & 3 & 235 & Multi-step heat treatment & 2 \\
\hline Algae & 1000 & 20 & 60 & 230 & - & 3 \\
\hline Tea Biomass & 1400 & 60 & 200 & 197 & $\begin{array}{l}\text { Washed with } 1 \mathrm{M} \mathrm{HCl}(5 \mathrm{~h}) \text { at } \\
60^{\circ} \mathrm{C} \text {, several washing steps }\end{array}$ & 4 \\
\hline Tire & 1100 & 20 & $\begin{array}{l}100 \\
600\end{array}$ & $\begin{array}{l}179 \\
154\end{array}$ & $\begin{array}{l}\text { Soaked in concentrated } \\
\qquad \mathrm{H}_{2} \mathrm{SO}_{4}\end{array}$ & 5 \\
\hline Peanut shell & 1100 & 20 & 150 & 308 & $\begin{array}{c}\text { Soaked in } 85 \% \mathrm{H}_{3} \mathrm{PO}_{4} \text { acid for } \\
3 \text { months }\end{array}$ & 6 \\
\hline $\begin{array}{l}\text { Boiled egg } \\
\text { yolks }\end{array}$ & 650 & 100 & $\begin{array}{c}1 \\
200\end{array}$ & $\begin{array}{l}208 \\
187\end{array}$ & Acid and alkali wash & 7 \\
\hline $\begin{array}{l}\text { Sorghum } \\
\text { Stalk }\end{array}$ & 1300 & 20 & 1 & $\begin{array}{l}245 \\
235 \\
\end{array}$ & $\begin{array}{l}\text { Washed by } 3 \mathrm{M} \mathrm{HCl} \text {, rinsed } \\
\text { by deionized water, filtration } \\
\text { and vacuum drying }\end{array}$ & 8 \\
\hline $\begin{array}{l}\text { Macshell } \\
\text { (this work) }\end{array}$ & 1100 & $\begin{array}{c}10 \\
20 \\
100\end{array}$ & $\begin{array}{c}3 \\
100 \\
300\end{array}$ & $\begin{array}{c}220 \\
180 \\
90\end{array}$ & No treatment & \\
\hline
\end{tabular}




\section{SI References:}

(1) Yamamoto, T.; Yamaguchi, T.; Nohira, T.; Hagiwara, R.; Fukunaga, A.; Sakai, S.; Nitta, K. Structural and Electrochemical Properties of Hard Carbon Negative Electrodes for Sodium Secondary Batteries Using the $\mathrm{Na}[\mathrm{FSA}]-[\mathrm{C} 3 \mathrm{C} 1 \mathrm{pyrr}][\mathrm{FSA}]$ Ionic Liquid Electrolyte. Electrochemistry 2017, 85 (7), 391-396. https://doi.org/10.5796/electrochemistry.85.391.

(2) Gomez-Martin, A.; Martinez-Fernandez, J.; Ruttert, M.; Winter, M.; Placke, T.; Ramirez-Rico, J. Correlation of Structure and Performance of Hard Carbons as Anodes for Sodium Ion Batteries. Chem. Mater. 2019, 31 (18), 7288-7299. https://doi.org/10.1021/acs.chemmater.9b01768.

(3) Meng, X.; Savage, P. E.; Deng, D. Trash to Treasure: From Harmful Algal Blooms to HighPerformance Electrodes for Sodium-Ion Batteries. Environ. Sci. Technol. 2015, 49 (20), 1254312550. https://doi.org/10.1021/acs.est.5b03882.

(4) Pei, L.; Cao, H.; Yang, L.; Liu, P.; Zhao, M.; Xu, B.; Guo, J. Hard Carbon Derived from Waste Tea Biomass as High-Performance Anode Material for Sodium-Ion Batteries. Ionics (Kiel). 2020, 26 (11), 5535-5542. https://doi.org/10.1007/s11581-020-03723-1.

(5) Li, Y.; Paranthaman, M. P.; Akato, K.; Naskar, A. K.; Levine, A. M.; Lee, R. J.; Kim, S.-O.; Zhang, J.; Dai, S.; Manthiram, A. Tire-Derived Carbon Composite Anodes for Sodium-Ion Batteries. J. Power Sources 2016, 316, 232-238. https://doi.org/10.1016/j.jpowsour.2016.03.071.

(6) Dou, X.; Hasa, I.; Saurel, D.; Jauregui, M.; Buchholz, D.; Rojo, T.; Passerini, S. Impact of the Acid Treatment on Lignocellulosic Biomass Hard Carbon for Sodium-Ion Battery Anodes. ChemSusChem 2018, 11 (18), 3276-3285. https://doi.org/10.1002/cssc.201801148.

(7) Guo, L.; An, Y.; Fei, H.; Feng, J.; Xiong, S.; Ci, L. Self-Templated Biomass-Derived Nitrogen-Doped Porous Carbons as High-Performance Anodes for Sodium Ion Batteries. Mater. Technol. 2017, 32 (10), 592-597. https://doi.org/10.1080/10667857.2017.1286555.

(8) Zhu, X.; Jiang, X.; Liu, X.; Xiao, L.; Cao, Y. A Green Route to Synthesize Low-Cost and HighPerformance Hard Carbon as Promising Sodium-Ion Battery Anodes from Sorghum Stalk Waste. Green Energy Environ. 2017, 2 (3), 310-315. https://doi.org/10.1016/j.gee.2017.05.004. 